\title{
TWIST1 and TWIST2 regulate glycogen storage and inflammatory genes in skeletal muscle
}

\author{
Jonathan M Mudry', Julie Massart', Ferenc L M Szekeres ${ }^{2}$ and Anna Krook ${ }^{1,2}$ \\ ${ }^{1}$ Section for Integrative Physiology, Department of Molecular Medicine and Surgery and \\ ${ }^{2}$ Section for Integrative Physiology, Department of Physiology and Pharmacology, Karolinska Institutet, \\ SE-171 77 Stockholm, Sweden
}

Correspondence should be addressed to A Krook

Email

Anna.Krook@ki.se

The authors and journal apologise for errors in the above paper, which appeared in volume 224 part 3, pages 303-313. The errors relate to the values given for high-density lipoprotein (HDL) and triglycerides (TG) in Table 1 on page 304.

The original Table 1 on page 304 stated that the mean ( \pm s.e.m) value for HDL in normal-glucose tolerant (NGT) human volunteers was $1.45 \pm 0.33 \mathrm{mmol} / \mathrm{l}$, that the value for TG in NGT human volunteers was $51.4 \pm 12.8 \mathrm{mmol} / \mathrm{l}$, and $92.5 \pm 16.9 \mathrm{mmol} / \mathrm{l}$ in type 2 diabetic (T2D) human volunteers.

The correct Table 1 should have stated that the mean ( \pm s.E.M) value for HDL in NGT human volunteers was $1.58 \pm 0.14 \mathrm{mmol} / \mathrm{l}$, that the value for TG in NGT human volunteers was $1.45 \pm 0.33 \mathrm{mmol} / 1$, and $1.24 \pm 0.11 \mathrm{mmol} / \mathrm{l} \mathrm{in}$ T2D human volunteers. The correct Table 1 is published in full below:

Table 1 Anthropometric measurements and metabolic parameters of human volunteers. Data are presented as means \pm S.E.M.

\begin{tabular}{|c|c|c|}
\hline & NGT & T2D \\
\hline$n$ & 10 & 10 \\
\hline $\operatorname{Sex}(F / M)$ & $5 / 5$ & $4 / 6$ \\
\hline Age (years) & $58 \pm 2$ & $63 \pm 1^{\dagger}$ \\
\hline Height (cm) & $171 \pm 3$ & $170 \pm 4$ \\
\hline Weight (kg) & $84.8 \pm 3.6$ & $90.8 \pm 5.8$ \\
\hline Waist circumference $(\mathrm{cm})$ & $93.2 \pm 2$ & $106.0 \pm 4^{\dagger}$ \\
\hline BMI $\left(\mathrm{kg} / \mathrm{m}^{2}\right)$ & $29.1 \pm 0.6$ & $31.3 \pm 1.2$ \\
\hline $\mathrm{SBP}(\mathrm{mmHg})$ & $133 \pm 5$ & $140 \pm 3$ \\
\hline $\mathrm{DBP}(\mathrm{mmHg})$ & $79 \pm 3$ & $78 \pm 2$ \\
\hline FBG (mmol/l) & $5.5 \pm 0.0$ & $7.9 \pm 0.2^{*}$ \\
\hline 2-h BG (mmol/l) & $6.6 \pm 0.4$ & $14.5 \pm 1.4^{\dagger}$ \\
\hline HbA1c (\%) & $4.6 \pm 0.1$ & $5.3 \pm 0.2^{\dagger}$ \\
\hline Insulin (pmol/l) & $51.4 \pm 12.5$ & $92.5 \pm 16.6^{\dagger}$ \\
\hline $\mathrm{HDL}(\mathrm{mmol} / \mathrm{l})$ & $1.58 \pm 0.14$ & $1.24 \pm 0.05^{\dagger}$ \\
\hline LDL (mmol/l) & $3.24 \pm 0.18$ & $2.74 \pm 0.28$ \\
\hline TG $(\mathrm{mmol} / \mathrm{l})$ & $1.45 \pm 0.33$ & $1.24 \pm 0.11$ \\
\hline IL6 (pg/ml) & $1.54 \pm 0.37$ & $2.23 \pm 0.51$ \\
\hline HOMA-IR & $1.97 \pm 0.10$ & $3.48 \pm 0.30$ * \\
\hline
\end{tabular}

NGT, normal-glucose tolerant; T2D, type 2 diabetes; SBP, systolic blood pressure; DBP, diastolic blood pressure; FBG, fasting blood glucose; HbA1C, glycated hemoglobin; TG, triglycerides; HOMA, homeostatic model assessment of insulin resistance. ${ }^{*} P<0.01 ;{ }^{\dagger} P<0.05$. 